\title{
On Batalin-Vilkovisky Formalism of Non-Commutative Field Theories
}

\author{
Klaus Bering ${ }^{a}$ and Harald Grosse ${ }^{b}$ \\ ${ }^{a}$ Institute for Theoretical Physics \& Astrophysics \\ Masaryk University \\ Kotlářská 2 \\ CZ-611 37 Brno \\ Czech Republic \\ ${ }^{b}$ Department of Physics \\ University of Vienna \\ Boltzmanngasse 5 \\ A-1090 Vienna \\ Austria
}

October 5, 2018

\begin{abstract}
We apply the BV formalism to non-commutative field theories, introduce BRST symmetry, and gauge-fix the models. Interestingly, we find that treating the full gauge symmetry in non-commutative models can lead to reducible gauge algebras. As one example we apply the formalism to the Connes-Lott two-point model. Finally, we offer a derivation of a superversion of the Harish-Chandra-Itzykson-Zuber integral.
\end{abstract}

PACS number(s): 02.40.Gh; 03.65.Ca; 11.10.-z; 11.10.Gh; 11.10.Nx; 11.15.-q. Keywords: Batalin-Vilkovisky Field-Antifield Formalism; Non-Commutative Geometry; Non-Commutative Field Theory; Matrix Models; Connes-Lott Model; Renormalization; Harish-Chandra-Itzykson-Zuber Integral.

${ }^{a} \mathrm{E}$-mail: bering@physics.muni.cz

${ }^{b} \mathrm{E}$-mail: harald.grosse@univie.ac.at 


\section{Introduction}

Developments around field theory models defined over non-commutative spaces are impressive. The formulation of various kinds of models is possible and was especially boosted after the paper [1]. The main hope to cure the diseases of quantum field theory was, however, only partially fulfilled. The canonical deformation leads to the IR/UV mixing.

For a non-commutative scalar field theory a detailed rigorous treatment of R. Wulkenhaar and one of the authors (H.G.) led to the identification of four relevant/marginal operators and a renormalizability proof [2]. The resulting model has the nice feature that the beta-function of the coupling constant vanishes to all orders of perturbation theory, which may lead to a constructive procedure [3, 4]. For a beautiful review of this subject with many references, see $[5]$.

Non-commutative gauge models have been treated first by expanding in the deformation parameter and using the Seiberg-Witten map [6, 7]. The treatments without expansions are extensive, but the question of renormalizability of these gauge models has been answered only partially, see, e.g., the proposals [8, 9] resulting from a heat kernel expansion. In addition, a Becchi-Rouet-Stora-Tyutin (BRST) approach was developed for a specific model [10] such that all propagators have nice decay properties resulting from a coupling to an oscillator term. Loop calculations in this specific model indicate improvements over elder models, but no conclusion for renormalization up to all orders has been possible. There has also been a recent attempt of using a different type of non-local counter-term in [11. In this way it is possible to yield what is called localization, see [12] for a recent treatment, but even this approach is still not conclusive.

Many of these non-commutative systems are matrix models with a cutoff given by the matrix size. Removing the cutoff leads to infinite gauge volume for gauge models. Therefore it is necessary to gauge-fix before taking the infinite matrix limit. This led us to study gauge models on matrix algebras including gauge-fixing, which is the main topic of this letter. We find that the Batalin-Vilkovisky (BV) formalism [13, 14] is here a useful (and in many instances a necessary) tool.

The letter is organized as follows. In Section 2, we discuss a construction of a non-commutative de Rham differential that works both for Heisenberg algebra type and Lie algebra type of noncommutativity. In Sections 36 we formulate non-commutative gauge theories in the BRST and the BV formalism. The gauge algebra can be reducible [14, 15], but gauge-fixing is still possible. In Section 9 we apply the stage-one reducible BV formalism to the Connes-Lott noncommutative model [16], which has built in the Higgs effect. Finally, in Appendix A] we give a localization argument for the superversion of the Harish-Chandra-Itzykson-Zuber integral.

We expect that the Batalin-Vilkovisky formalism can be applied to many other models of noncommutative quantum field theory, particularly when analyzing renormalizability, and we shall consider more applications in the future.

General Remarks About Notation: Adjectives from super-mathematics such as "graded", "super", etc., are implicitly implied. The commutator $[f, g]$ of two non-commutative 
forms $f$ and $g$, of Grassmann-parity $\varepsilon_{f}, \varepsilon_{g}$ and of form-degree $p_{f}, p_{g}$, is defined as

$$
[f, g]=f g-(-1)^{\varepsilon_{f} \varepsilon_{g}+p_{f} p_{g}} g f
$$

There is a tradition in quantum mechanical textbooks to put a hat " $\wedge$ " on top of a noncommutative operator $\hat{f}$, to distinguish it from its commutative symbol $f$, which is just a function. However, we shall not write hats " $\wedge$ " to avoid clutter. The commutative symbol will only appear in eqs. (7.1), (7.3) and (7.4) below.

Finally, we should mention that we do often not discuss reality/Hermiticity conditions explicitly. Since we will often have no explicit factors of the imaginary unit $\sqrt{-1}$ in our formulas, we should warn that the variables are sometimes implicitly assumed to be imaginary/anti-Hermitian rather than real/Hermitian.

\section{Non-Commutative de Rham Differential}

Let there be given an associative algebra $\mathcal{A}$ with algebra generators $x_{\mu}, \mu \in I$, and a unit $\mathbf{1}$. It is assumed that the set $\{\mathbf{1}\} \cup\left\{x_{\mu} \mid \mu \in I\right\}$ consists of linearly independent elements. Physically, we can think of the algebra $\mathcal{A}$ as a non-commutative world volume with non-commutative coordinates $x_{\mu}$. We will often realize the $x_{\mu}$ coordinates as matrices $\left(x_{\mu}\right)^{a}{ }_{b}$, where the matrix index " $a$ " carries Grassmann-parity $\varepsilon_{a}$, so that the matrix entry $\left(x_{\mu}\right)^{a}{ }_{b}$ has Grassmann-parity

$$
\varepsilon\left(\left(x_{\mu}\right)_{b}^{a}\right)=\varepsilon_{\mu}+\varepsilon_{a}+\varepsilon_{b}
$$

We will also assume that there exists a cyclic trace operation "tr" for the algebra $\mathcal{A}$. The trace operation "tr" may be thought of as an integration over the non-commutative world volume. In a matrix realization, the trace "tr" is the supertrace,

$$
\operatorname{tr}\left(x_{\mu}\right)=(-1)^{\varepsilon_{a}\left(\varepsilon_{\mu}+1\right)}\left(x_{\mu}\right)^{a}{ }_{a} .
$$

We next assume that the commutator $\left[x_{\mu}, x_{\nu}\right]$ of two coordinates $x_{\mu}$ and $x_{\nu}$ is a linear combination of $\{\mathbf{1}\} \cup\left\{x_{\mu} \mid \mu \in I\right\}$, i.e., that there exists antisymmetric structure constants

$$
\begin{aligned}
\theta_{\mu \nu} & =-(-1)^{\varepsilon_{\mu} \varepsilon_{\nu}} \theta_{\nu \mu}, \\
f_{\mu \nu}{ }^{\lambda} & =-(-1)^{\varepsilon_{\mu} \varepsilon_{\nu}} f_{\nu \mu}{ }^{\lambda}
\end{aligned}
$$

such that

$$
\left[x_{\mu}, x_{\nu}\right]=\theta_{\mu \nu} \mathbf{1}+f_{\mu \nu}{ }^{\lambda} x_{\lambda} .
$$

This will cover two main applications: the Heisenberg algebra, i.e., the constant case with $f_{\mu \nu}{ }^{\lambda}=0$; and the Lie algebra, i.e., the linear case with $\theta_{\mu \nu}=0$. The Jacobi identity for commutator $[\cdot, \cdot]$ and the linear independence imply that

$$
\begin{aligned}
\sum_{\text {cycl. } \mu, \nu, \lambda}(-1)^{\varepsilon_{\mu} \varepsilon_{\lambda}} f_{\mu \nu}{ }^{\kappa} \theta_{\kappa \lambda} & =0 \\
\sum_{\text {cycl. } \mu, \nu, \lambda}(-1)^{\varepsilon_{\mu} \varepsilon_{\lambda}} f_{\mu \nu}{ }^{\kappa} f_{\kappa \lambda}{ }^{\rho} & =0
\end{aligned}
$$


One next defines a (not necessarily nilpotent) Bosonic de Rham one-form

$$
\Omega=c^{\mu} x_{\mu}+\frac{1}{2} c^{\nu} c^{\mu} f_{\mu \nu}{ }^{\lambda} b_{\lambda} .
$$

Here the $c^{\mu}$ 's and the $b_{\mu}$ 's are bases for one-forms and minus-one-forms(=vector fields), respectively.

$$
\left[b_{\mu}, c^{\nu}\right]=\delta_{\mu}^{\nu},
$$

and all other commutators vanish. The form degree " $p$ " can be thought of as a world volume ghost degree, and in this sense, the $c^{\mu}$ 's and the $b_{\mu}$ 's are world volume ghosts and ghost momenta. (This should not be confused with the actual ghost number "gh", which lives in a target space.)

The components $\Omega_{\mu}$ of the de Rham one-form $\Omega=c^{\mu} \Omega_{\mu}$ is

$$
\Omega_{\mu}=x_{\mu}+\frac{1}{2} c^{\nu} f_{\nu \mu}{ }^{\lambda} b_{\lambda} .
$$

The square

$$
\Omega^{2}=\frac{1}{2}[\Omega, \Omega]=-\frac{1}{2} c^{\nu} c^{\mu} \theta_{\mu \nu}=\frac{1}{2} c^{\mu} \theta_{\mu \nu} c^{\nu}(-1)^{\varepsilon_{\nu}}
$$

of the de Rham one-form $\Omega$ is a (not necessarily vanishing) two-form. The non-commutative exterior de Rham differential $d$ is now implemented as

$$
d:=[\Omega, \cdot] .
$$

The square

$$
d^{2}=[\Omega,[\Omega, \cdot]]=\left[\Omega^{2}, \cdot\right]
$$

of the de Rham differential " $d$ " vanishes on elements $F=F(x, c) \in \Omega^{\bullet}(\mathcal{A})$ that do not depend on the minus-one-forms $b_{\mu}$.

\section{Non-Commutative Gauge Field Models}

For these models it is possible to introduce a one-form valued covariant derivative

$$
\nabla=\Omega+A=c^{\mu} \nabla_{\mu},
$$

where the one-form $A=c^{\mu} A_{\mu}$ is a gauge potential. One usually assumes that the gauge field components $A_{\mu}=A_{\mu}(x)$ do not depend on the $c$ 's and $b$ 's. The components $\nabla_{\mu}$ of the covariant derivative $\nabla$ are

$$
\nabla_{\mu}=\Omega_{\mu}+A_{\mu}=X_{\mu}+\frac{1}{2} c^{\nu} f_{\nu \mu}{ }^{\lambda} b_{\lambda},
$$

where

$$
X_{\mu}:=x_{\mu}+A_{\mu}
$$

are the covariant coordinates. One can think of $X_{\mu}=X_{\mu}(x)$ as coordinates on a target space. The field strength $F$ and the curvature $R$ are defined as

$$
\begin{aligned}
& F:=(d A)+A^{2}=-\frac{1}{2} c^{\nu} c^{\mu} F_{\mu \nu}=\frac{1}{2} c^{\mu} F_{\mu \nu} c^{\nu}(-1)^{\varepsilon_{\nu}} \\
& R:=\nabla^{2}=\frac{1}{2}[\nabla, \nabla]=\Omega^{2}+F=-\frac{1}{2} c^{\nu} c^{\mu} R_{\mu \nu}=\frac{1}{2} c^{\mu} R_{\mu \nu} c^{\nu}(-1)^{\varepsilon_{\nu}},
\end{aligned}
$$


Table 1: Parities, degrees and ghost numbers of various objects.

\begin{tabular}{|c|c|c|c|c|}
\hline & & $\begin{array}{l}\text { Grass- } \\
\text { mann } \\
\text { parity }\end{array}$ & $\begin{array}{c}\text { World } \\
\text { volume } \\
\text { form } \\
\text { degree } \\
\end{array}$ & $\begin{array}{c}\text { Target } \\
\text { space } \\
\text { ghost } \\
\text { number }\end{array}$ \\
\hline & $\downarrow$ Symbol $\rightarrow$ & $\varepsilon$ & $p$ & gh \\
\hline World volume coordinate & $x_{\mu}$ & $\overline{\varepsilon_{\mu}}$ & $\overline{0}$ & 0 \\
\hline World volume one-form & $c^{\mu}$ & $\varepsilon_{\mu}$ & 1 & 0 \\
\hline World volume minus-one-form & $b_{\mu}$ & $\varepsilon_{\mu}$ & -1 & 0 \\
\hline De Rham one-form & $\Omega=c^{\mu} \Omega_{\mu}$ & 0 & 1 & 0 \\
\hline De Rham differential & $d=[\Omega, \cdot]$ & 0 & 1 & 0 \\
\hline General target space field & $\Phi^{\alpha}$ & $\bar{\varepsilon}_{\alpha}$ & 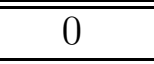 & $\mathrm{gh}_{\alpha}$ \\
\hline Target space coordinate & $X_{\mu}=x_{\mu}+A_{\mu}$ & $\varepsilon_{\mu}$ & 0 & 0 \\
\hline Gauge parameter & $\Xi$ & 0 & 0 & 0 \\
\hline Target space ghost & $C$ & 1 & 0 & 1 \\
\hline Target space antighost & $\bar{C}$ & 1 & 0 & -1 \\
\hline Lagrange multiplier & $\Pi$ & 0 & 0 & 0 \\
\hline Gauge condition & $\chi$ & 0 & 0 & 0 \\
\hline Ghost-for-ghost & $\eta$ & 0 & 0 & 2 \\
\hline Antighost-for-ghost & $\bar{\eta}$ & 0 & 0 & -2 \\
\hline Lagr.-mult.-for-ghost & $\overline{\bar{\pi}}$ & 1 & 0 & -1 \\
\hline Extra ghost & $\widetilde{\eta}$ & 0 & 0 & 0 \\
\hline Extra Lagrange multiplier & $\widetilde{\pi}$ & 1 & 0 & 1 \\
\hline General target space antifield & $\Phi_{\alpha}^{*}$ & $\varepsilon_{\alpha}+1$ & 0 & $-1-\operatorname{gh}_{\alpha}$ \\
\hline Coordinate antifield & $X^{\mu *}$ & $\varepsilon_{\mu}+1$ & 0 & -1 \\
\hline Ghost antifield & $C^{*}$ & 0 & 0 & -2 \\
\hline Antighost antifield & $\bar{C}^{*}$ & 0 & 0 & 0 \\
\hline Lagrange multiplier antifield & $\Pi^{*}$ & 1 & 0 & -1 \\
\hline "Ghost-for-ghost antifield & 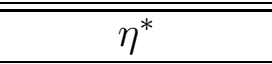 & 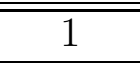 & 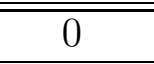 & -3 \\
\hline Antighost-for-ghost antifield & $\bar{\eta}^{*}$ & 1 & 0 & 1 \\
\hline Lagr.-mult.-for-ghost antifield & $\bar{\pi}^{*}$ & 0 & 0 & 0 \\
\hline Extra ghost antifield & $\widetilde{\eta}^{*}$ & 1 & 0 & -1 \\
\hline Extra Lagr.mult. antifield & $\tilde{\pi}^{*}$ & 0 & 0 & -2 \\
\hline Classical BRST operator & $\overline{\mathbf{s}=(S, \cdot)}$ & $\overline{1} 1$ & $\overline{0}$ & 1 \\
\hline Odd Laplacian & $\Delta$ & 1 & 0 & 1 \\
\hline Gauge-fermion & $\Psi$ & 1 & 0 & -1 \\
\hline
\end{tabular}


respectively. Their components $F_{\mu \nu}$ and $R_{\mu \nu}$ do not depend on the c's and b's.

$$
\begin{aligned}
& F_{\mu \nu}=\left[x_{\mu}, A_{\nu}\right]+\left[A_{\mu}, x_{\nu}\right]+\left[A_{\mu}, A_{\nu}\right]-f_{\mu \nu}{ }^{\lambda} A_{\lambda}, \\
& R_{\mu \nu}=F_{\mu \nu}+\theta_{\mu \nu}=\left[X_{\mu}, X_{\nu}\right]-f_{\mu \nu}{ }^{\lambda} X_{\lambda} .
\end{aligned}
$$

The typical starting action $S_{0}$ is of the form $S_{0}=\operatorname{tr} L_{0}(X)$, where $L_{0}=L_{0}(X)$ is a polynomial in the $X_{\mu}$ 's. The covariant coordinates $X_{\mu}$ transform as $X_{\mu} \rightarrow X_{\mu}^{g}=g^{-1} X_{\mu} g$ under gauge transformations $g=e^{\Xi}$. Therefore the infinitesimal gauge transformations takes the form

$$
\delta X_{\mu}=\left[X_{\mu}, \Xi\right]=-\left[\Xi, X_{\mu}\right]
$$

where $\Xi \in \mathcal{A}$ is the infinitesimal gauge parameter. Obviously, $F_{\mu \nu}$ and $R_{\mu \nu}$ transform covariantly as well. Note that the matrix entries $\Xi_{b}^{a}$ of the gauge parameter matrix $\Xi$ need not be independent, see the Hermitian one-matrix model in Section 8 for a simple example. In more complicated situations, it might not be possible to identify (or, for other reasons, not desirable to work with) an independent set of gauge generators. In that case one would have to work with a reducible gauge algebra, and to introduce a new set of so-called stage-one gauge symmetries to handle the over-complete set of original gauge symmetries. In the BRST language this leads to ghosts-for-ghosts. For a simple example of a stage-one reducible gauge theory, see next Section 9. Nevertheless, we shall for the rest of this Section 3 for simplicity assume that it is possible to consistently pick an independent set of gauge parameters. It is then possible to encode the gauge symmetry (3.8) in a Fermionic nilpotent BRST operator $\mathbf{s}$ of the form

$$
\mathbf{s} X_{\mu}=(-1)^{\varepsilon_{\mu}}\left[X_{\mu}, C\right]=-\left[C, X_{\mu}\right], \quad \mathbf{s} C=-\frac{1}{2}[C, C]
$$

Here $C \in \mathcal{A}$ is the target space ghost. The BRST operator $\mathbf{s}$ is by definition extended to polynomials in $X_{\mu}$ and $C$ via a non-commutative Leibniz rule,

$$
\mathbf{s}(f g)=(\mathbf{s} f) g+(-1)^{\varepsilon_{f}} f(\mathbf{s} g)
$$

In other words, the BRST operator "s" is a Fermionic vector field on a non-commutative space. The square $\mathbf{s}^{2}=\frac{1}{2}[\mathbf{s}, \mathbf{s}]$ of the BRST operator is again a vector field, which satisfies a non-commutative Leibniz rule $\mathbf{s}^{2}(f g)=\left(\mathbf{s}^{2} f\right) g+f\left(\mathbf{s}^{2} g\right)$, and is in fact identical to zero,

$$
\mathbf{s}^{2}=0 \text {. }
$$

\section{BV Odd Laplacian and Antibracket}

The BRST formulation can be further encoded into the BV formalism [13, 14]. If the gauge transformations form a reducible or an open gauge algebra, this step will often be necessary. The original BV recipe (which is formulated in terms of supercommutative field variables $\phi^{\alpha}(x)$ in a path integral setting) can be directly applied without modifications to non-commutative fields $\Phi^{\alpha}$ (where $\Phi^{\alpha}$ is a collective notation for all fields $\Phi^{\alpha}=\left\{X_{\mu}, C, \ldots\right\}$ ) simply by treating the matrix entries $\left(\Phi^{\alpha}\right)_{b}^{a}$ (which are supercommutative objects!) as the fundamental variables. For instance, the odd Laplacian is

$$
\Delta:=(-1)^{\varepsilon\left(\left(\Phi^{\alpha}\right)^{a}{ }_{b}\right)} \frac{\overrightarrow{\partial^{\ell}}}{\partial\left[\left(\Phi^{\alpha}\right)_{b}^{a}{ }_{b}\right]} \frac{\overrightarrow{\partial^{\ell}}}{\partial\left[\left(\Phi_{\alpha}^{*}\right)^{b}{ }_{a}\right]},
$$


where $\Phi_{\alpha}^{*}$ are the corresponding matrix-valued antifields. (We assume for simplicity that the matrices $\Phi^{\alpha}$ are world volume zero-forms.) The antibracket reads

$$
(F, G):=F\left(\frac{\overleftarrow{\partial^{r}}}{\partial\left[\left(\Phi^{\alpha}\right)_{b}^{a}\right]} \frac{\overrightarrow{\partial^{\ell}}}{\partial\left[\left(\Phi_{\alpha}^{*}\right)_{a}^{b}\right]}-\frac{\overleftarrow{\partial^{r}}}{\partial\left[\left(\Phi_{\alpha}^{*}\right)_{b}^{a}\right]} \frac{\overrightarrow{\partial^{\ell}}}{\partial\left[\left(\Phi^{\alpha}\right)^{b}{ }_{a}\right]}\right) G
$$

In particular, the antibrackets of fundamental variables read

$$
\left(\left(\Phi^{\alpha}\right)_{b}^{a},\left(\Phi_{\beta}^{*}\right)_{d}^{c}\right)=\delta_{\beta}^{\alpha} \delta_{d}^{a} \delta_{b}^{c}, \quad\left(\left(\Phi^{\alpha}\right)_{b}^{a},\left(\Phi^{\beta}\right)_{d}^{c}\right)=0, \quad\left(\left(\Phi_{\alpha}^{*}\right)_{b}^{a},\left(\Phi_{\beta}^{*}\right)_{d}^{c}\right)=0 .
$$

Let us mention that the set $\mathcal{M}$ of points $\Gamma^{A} \equiv\left(\Phi^{\alpha} ; \Phi_{\alpha}^{*}\right)$ is called the antisymplectic phase space. The antibracket $(\cdot, \cdot)$ is an antisymplectic structure on this phase space $\mathcal{M}$.

REMARK: If one draws the index structure of a trace as a loop, then the antibracket $(F, G)$ always joints two index loops $F=\operatorname{tr} f\left(\Phi, \Phi^{*}\right)$ and $G=\operatorname{tr} g\left(\Phi, \Phi^{*}\right)$ into a single index loop. The action of the antibracket $(\cdot, \cdot)$ on multiple loops can be determined via Leibniz rule

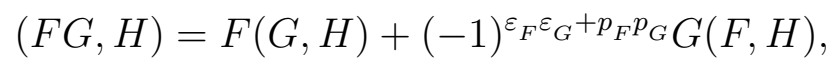

so that in general

$$
(\underbrace{\bigcirc \bigcirc \cdots \bigcirc}_{n \text { loops }}, \underbrace{\bigcirc \bigcirc \cdots \bigcirc}_{m \text { loops }})=\sum \underbrace{\bigcirc \bigcirc \cdots \bigcirc \bigcirc \bigcirc}_{n+m-1 \text { loops }}
$$

The odd Laplacian $\Delta$ adds an extra index loop $\Delta F$ when applied to a single trace $F=\operatorname{tr} f\left(\Phi, \Phi^{*}\right)$,

$$
\Delta(\underbrace{\bigcirc}_{1 \text { loop }})=\sum \underbrace{\bigcirc \bigcirc}_{2 \text { loops }}
$$

The action of $\Delta$ on multiple loops can be determined from the formula

$$
\Delta(F G)=(\Delta F) G+(-1)^{\varepsilon_{F}}(F, G)+(-1)^{\varepsilon_{F}} F(\Delta G),
$$

so that in general for $n \geq 2$,

$$
\Delta(\underbrace{\bigcirc \bigcirc \cdots \bigcirc}_{n \text { loops }})=\sum \underbrace{\bigcirc \bigcirc \cdots \bigcirc}_{n+1 \text { loops }}+\sum \underbrace{\bigcirc \cdots \bigcirc}_{n-1 \text { loops }} \text {. }
$$

This picture superficially resembles the loop operator of Chas-Sullivan in string topology [17], and the handle operator of Zwiebach in closed string field theory [18], mostly because all the mentioned cases are governed by their underlying Batalin-Vilkovisky algebras.

\section{BV Proper Action}

In the BV scheme [13, 14] one searches for a proper action $S$ to the classical master equation

$$
(S, S)=0
$$


In the above class of models, the minimal proper master action $S$ is given by $S=\operatorname{tr} L$, where the Lagrangian density $L$ is

$$
L=L_{0}+(-1)^{\varepsilon_{\mu}} X^{\mu *} \mathbf{s} X_{\mu}-C^{*} \mathbf{s} C \approx L_{0}-\left(\mathbf{s} X_{\mu}\right) X^{\mu *}-(\mathbf{s} C) C^{*},
$$

and where $X^{\mu *} \in \mathcal{A}$ and $C^{*} \in \mathcal{A}$ are the corresponding antifields, and " $\approx$ " means equality modulo total commutator terms. The antifields are generators of BRST symmetry. The classical BRST operator in the $\mathrm{BV}$ formalism is $\mathbf{s}=(S, \cdot)$. In general, there could be quantum corrections to the classical master action $S$. However, quantum corrections are not needed if $\Delta S=0$, which is true for the action (5.2).

REMARK: Note that the BRST operator "s" acts on a whole matrix $\Phi^{\alpha}$ versus a matrix entry $\left(\Phi^{\alpha}\right)_{b}^{a}$ according to the rule

$$
\mathbf{s}\left[\left(\Phi^{\alpha}\right)_{b}^{a}\right]=(-1)^{\varepsilon_{a}}\left(\mathbf{s} \Phi^{\alpha}\right)^{a}{ }_{b}
$$

This sign factor (5.3) is due to a permutation of the row-index " $a$ " and BRST operator "s". (Recall that the matrix entries $\left(\Phi^{\alpha}\right)^{a}{ }_{b}$ of a supermatrix $\Phi^{\alpha}$ should strictly speaking be written as $\left.{ }^{a}\left(\Phi^{\alpha}\right)_{b}.\right)$ For a similar reason, if one identifies $\delta \leftrightarrow \mu \mathbf{s}$ and $\Xi \leftrightarrow \mu C$ in eqs. (3.8) and (3.9), where $\mu$ is a Fermionic parameter, then the matrix entries should be identified as $\Xi_{b}^{a} \leftrightarrow(-1)^{\varepsilon_{a}} \mu C^{a}{ }_{b}$.

\section{BV Gauge-Fixing}

The standard BV procedure to gauge-fix is to extend the Lagrangian density $L$ with a nonminimal sector $L \rightarrow L+\bar{C}^{*} \Pi$, where $\bar{C} \in \mathcal{A}$ is an antighost and $\Pi \in \mathcal{A}$ is a Lagrange multiplier, and $\bar{C}^{*}, \Pi^{*} \in \mathcal{A}$ are the corresponding antifields. In the end, all the antifields $\Phi_{\alpha}^{*}$ are replaced

$$
\left(\Phi_{\alpha}^{*}\right)_{b}^{a} \longrightarrow \frac{\partial \Psi}{\partial\left[\left(\Phi^{\alpha}\right)_{a}^{b}\right]},
$$

where $\Psi=\Psi(\Phi)$ is a gauge fermion. It was proved in the original work [13, 14] that the partition function $\mathcal{Z}$ is perturbatively well-defined and will locally not depend on the gauge-fermion $\Psi$ as long as the quantum master equation holds, and the action and $\Psi$ satisfies certain rank conditions. Usually $\Psi$ is taken of the form

$$
\Psi=\operatorname{tr}(\bar{C} \chi),
$$

where $\chi \in \mathcal{A}$ is the gauge-fixing condition. One possible gauge is a Lorenz type gauge

$$
\chi=\left[n^{\mu}, X_{\mu}\right]
$$

where $n^{\mu} \in \mathcal{A}$ is a fixed vector. Gauge-fixing can be considerably generalized, see Ref. [19].

\section{Star Product}

Instead of matrices, it is also popular to formulate non-commutative field theories in terms of fields $\phi^{\alpha}(x)$ (so-called symbols) and an associative star product " $*$ ", which is often taken to be 
of the Groenewold-Moyal type

$$
(f * g)(x)=f(x) \exp \left[\frac{\overleftarrow{\partial^{r}}}{\partial x_{\mu}} m_{\mu \nu} \frac{\overrightarrow{\partial^{\ell}}}{\partial x_{\nu}}\right] g(x) .
$$

The Groenewold-Moyal star product (7.1) corresponds to the case, where the structure constants in eq. (2.5) yield a Heisenberg algebra,

$$
f_{\mu \nu}^{\lambda}=0, \quad \theta_{\mu \nu}=m_{\mu \nu}-(-1)^{\varepsilon_{\mu} \varepsilon_{\nu}} m_{\nu \mu} .
$$

Batalin-Vilkovisky formalism also works in this setting [20, 21] (since the symbols are supercommutative!), and considerations of local BRST cohomology [22] have been extended to non-commutative field theories [23], at least when using the pragmatic definition of locality. The pragmatic definition of a local functional

$$
F=\int d x f(x)
$$

is an integral over a function

$$
f(x)=f\left(\phi(x), \partial \phi(x), \ldots, \partial^{N} \phi(x), x\right)
$$

that depends locally on the fields $\phi^{\alpha}(x)$ in the point $x$ and its derivatives to some finite order $N$. The corresponding definition of a local functional $F$ in a matrix-setting is, roughly speaking, a single-trace

$$
F=\operatorname{tr} f(\Phi),
$$

where $f=f(\Phi)$ is a polynomial in the $\Phi^{\alpha}$ 's. It could be interesting to investigate local BRST cohomology from this matrix-point-of-view.

\section{Hermitian One-Matrix Model}

Consider a Hermitian one-matrix model $L_{0}(H)=\sum_{n} a_{n} H^{n} / n$ !, where $X=H$ is a Bosonic Hermitian endomorphism in a $\left(N_{0} \mid N_{1}\right)$ super vector space $V$ of dimension $N=N_{0}+N_{1}$, and where the $a_{n}$ 's are Bosonic numbers. The original action $S_{0}=\operatorname{tr} L_{0}$ is invariant under gauge transformations $H \rightarrow H^{g}=g^{-1} H g$, where $g=e^{\Xi} \in U\left(N_{0} \mid N_{1}\right)$. The model has $N^{2}$ gauge parameters $\Xi_{b}^{a}$ corresponding to the number of matrix entries in $H$. However, the Bosonic eigenvalues $\lambda_{1}, \lambda_{2}, \ldots, \lambda_{N}$, of $X$ are $N$ gauge-invariant quantities, which cannot be changed by gauge transformations of adjoint type. Hence there are actually only $N(N-1)$ independent gauge parameters. Thus the gauge algebra is reducible.

For a diagonal matrix $H$, the $N$ redundant gauge parameters may be identified with the diagonal matrix entries $\Xi_{1}^{1}, \Xi_{2}^{2}, \ldots, \Xi^{N}$, at the infinitesimal level. It is possible to truncate the reducible gauge algebra to a stage-zero irreducible gauge algebra as follows. Since all Hermitian matrices $H$ are diagonalizable, it is always possible to pick a diagonal gauge. We implement the diagonal gauge via a Lorenz type gauge condition

$$
\chi=[n, H], \quad n=\operatorname{diag}\left(\nu_{1}, \ldots, \nu_{N}\right), \quad \chi_{b}^{a}=\left(\nu_{a}-\nu_{b}\right) H_{b}^{a},
$$


where $n$ is a fixed diagonal matrix with different eigenvalues $\nu_{1}, \nu_{2}, \ldots, \nu_{N}$. Since there are only $N(N-1)$ independent gauge symmetries, the ghost $C$ and antighost $\bar{C}$ have only off-diagonal entries. There are also only be $N(N-1)$ off-diagonal $\chi_{b}^{a}$ gauge conditions (8.1) to implement, $1 \leq a \neq b \leq N$, so the Lagrange multiplier $\Pi$ contains only off-diagonal entries as well.

The antifields $\Phi^{*}=\partial \Psi / \partial \Phi$ with $\Psi=\operatorname{tr}(\bar{C} \chi)$ become

$$
H^{*}=[\bar{C}, n], \quad C^{*}=0, \quad \bar{C}^{*}=\chi, \quad \Pi^{*}=0 .
$$

The gauge-fixed action (5.2) reads

$$
\begin{aligned}
& \left.S\right|_{\Phi^{*}=\frac{\partial \Psi}{\partial \Phi}} \sim S_{0}+\quad \operatorname{tr}(\bar{C}[n,[H, C]])+\operatorname{tr}([n, H] \quad \Pi) \\
& \sim S_{0}+\sum_{a \neq b}(-1)^{\varepsilon_{b}}\left[\bar{C}_{a}^{b}\left(\nu_{a}-\nu_{b}\right)\left(H_{a}^{a}{ }_{a}-H_{b}^{b}\right) C_{b}^{a}+\left(\nu_{b}-\nu_{a}\right) H_{a}^{b} \quad \Pi_{b}^{a}\right] . \\
& \text { Gauge- Orig. Faddeev- Gauge Lagr. } \\
& \text { fixed ac- Popov condi- mult. } \\
& \text { action tion matrix tion }
\end{aligned}
$$

The partition function $\mathcal{Z}$ becomes

$$
\begin{aligned}
\mathcal{Z} & =\int[d H][d C][d \bar{C}][d \Pi] e^{\frac{i}{\hbar} S\left(\Phi, \Phi^{*}=\frac{\partial \Psi}{\partial \Phi}\right)} \\
& \sim \int d \lambda_{1} \cdots d \lambda_{N} e^{\frac{i}{\hbar} \operatorname{tr} L_{0}\left(\operatorname{diag}\left(\lambda_{1}, \ldots, \lambda_{N}\right)\right)} \Delta^{2}\left(\lambda_{a}\right)
\end{aligned}
$$

up to a numerical factor, where the super-Vandermonde determinant is

$$
\Delta\left(\lambda_{a}\right)=\prod_{1 \leq a<b \leq N}\left(\lambda_{b}-\lambda_{a}\right)^{\left[(-1)^{\varepsilon_{a}+\varepsilon_{b}}\right]}
$$

The result (8.3) is manifestly independent of the gauge-fixing parameters $\nu_{1}, \nu_{2}, \ldots, \nu_{N}$, as it should be. The integrand consists of a classical Boltzmann factor times a square $\Delta^{2}\left(\lambda_{a}\right)$ of a Vandermonde superdeterminant, whose $N(N-1)$ factors reflect the $N(N-1)$ independent gauge symmetries.

The above removal of the $N$ diagonal gauge parameters directions $\Xi_{a}^{a}=0, a \in\{1,2, \ldots, N\}$, can also be seen as a way to get rid of $N$ zero-modes in the Faddeev-Popov determinant (if one assumes that all the eigenvalues $\lambda_{1}, \lambda_{2}, \ldots, \lambda_{N}$, are different).

\section{Connes-Lott Model for a 2-Point Space}

The algebra $\mathcal{A}=\operatorname{End}(V)$ of the Connes-Lott model [16] consists of endomorphisms in a $(1 \mid 1)$ super vector space $V$, i.e., the vector space $V$ has one Bosonic and one Fermionic direction. One may think of the endomorphisms as $2 \times 2$ matrices. We will for simplicity only consider matrices that are either diagonal or off-diagonal and that carry definite Grassmann-parity. Note that 
diagonal and off-diagonal matrices (with matrix entries of the same Grassmann-parity) carry opposite Grassmann-parity.

The Connes-Lott model for a 2 -point space has only one algebra generator $x_{1}$ and one covariant coordinate $X_{1}=x_{1}+A_{1}$. They are off-diagonal Fermionic matrices

$$
x_{1}=\left(\begin{array}{cc}
0 & 1 \\
1 & 0
\end{array}\right), \quad X_{1}=\left(\begin{array}{cc}
0 & H \\
\bar{H} & 0
\end{array}\right),
$$

where $H$ is a complex-valued Bosonic Higgs field, and $\bar{H}$ is the complex conjugated field. The single world volume coordinate $x_{1}$ is a non-commutative coordinate,

$$
\left[x_{1}, x_{1}\right]=\theta_{11} \mathbf{1}, \quad \theta_{11}=2 .
$$

The original action $S_{0}=\operatorname{tr} L_{0}$ is given as

$$
L_{0} \sim F_{11} F^{11} \Gamma=\frac{1}{4}\left(F_{11}\right)^{2} \Gamma=\left(|H|^{2}-1\right)^{2} \Gamma,
$$

where

$$
F_{11}=\left[x_{1}, A_{1}\right]+\left[A_{1}, x_{1}\right]+\left[A_{1}, A_{1}\right]=\left[X_{1}, X_{1}\right]-\left[x_{1}, x_{1}\right]=2\left(|H|^{2}-1\right) \mathbf{1},
$$

and where $\Gamma$ is a chirality operator,

$$
\Gamma:=\left(\begin{array}{cc}
1 & 0 \\
0 & -1
\end{array}\right)
$$

The chirality operator $\Gamma$ breaks down a $U(1 \mid 1)$ supergroup (which naturally acts on the $(1 \mid 1)$ vector space $V$ ) to a diagonal $U(1) \times U(1)$ subgroup. In detail, the gauge group element $g \in U(1) \times U(1)$ is of the form

$$
g=e^{i \Xi}=\left(\begin{array}{cc}
e^{i \xi} & 0 \\
0 & e^{i \xi^{\prime}}
\end{array}\right)
$$

with gauge parameter

$$
\Xi=\left(\begin{array}{cc}
\xi & 0 \\
0 & \xi^{\prime}
\end{array}\right)
$$

The transformed covariant coordinate $X_{1}^{g}$ is

$$
X_{1}^{g}=g^{-1} X_{1} g=\left(\begin{array}{cc}
0 & H^{g} \\
H^{g} & 0
\end{array}\right), \quad H^{g}=H e^{-i \xi_{-}}, \quad \xi_{ \pm}:=\xi \pm \xi^{\prime} .
$$

The eigenvalues $\pm|H|$ of the matrix $X_{1}$ (and hence the modulus $|H|$ ) are preserved under gauge transformations, because they are just similarity transformations. The infinitesimal gauge transformation reads

$$
\delta X_{1}=i\left[X_{1}, \Xi\right], \quad \delta(\operatorname{Re}(H))=\xi_{-} \operatorname{Im}(H), \quad \delta(\operatorname{Im}(H))=-\xi_{-} \operatorname{Re}(H) .
$$

Clearly, the two $U(1)$ gauge factors are linearly dependent, i.e., they constitute a reducible gauge algebra. The gauge-for-gauge symmetry $\widetilde{\delta}$ is of the form

$$
\widetilde{\delta} \Xi=\left(\begin{array}{cc}
\widetilde{\delta} \xi & 0 \\
0 & \widetilde{\delta} \xi^{\prime}
\end{array}\right)=\left(\begin{array}{cc}
1 & 0 \\
0 & 1
\end{array}\right) \zeta, \quad \widetilde{\delta} \xi_{+}=2 \zeta, \quad \widetilde{\delta} \xi_{-}=0,
$$


where $\zeta$ is a gauge-for-gauge parameter. Although it is immediately clear that we can go to an irreducible basis by fixing $\xi_{+}=0$, let us here for illustrative purposes show how to treat the Connes-Lott 2-point model as a stage-one reducible gauge system [14]. 1

The Fermionic reducible ghost is

$$
C=\left(\begin{array}{cc}
c & 0 \\
0 & c^{\prime}
\end{array}\right)
$$

The BRST transformations are

$$
\begin{aligned}
& \left(\begin{array}{cc}
0 & \mathbf{s} H \\
-\mathbf{s} H & 0
\end{array}\right) \stackrel{5.3}{=} \mathbf{s} X_{1}=-i\left[X_{1}, C\right]=-i\left(\begin{array}{cc}
0 & H c_{+} \\
\bar{H} c_{+} & 0
\end{array}\right), \\
& \mathbf{s}(\operatorname{Re}(H))=c_{+} \operatorname{Im}(H), \quad \mathbf{s}(\operatorname{Im}(H))=-c_{+} \operatorname{Re}(H), \quad c_{ \pm}:=c \pm c^{\prime}, \\
& \left(\begin{array}{cc}
\mathbf{s} c & 0 \\
0 & -\mathbf{s} c^{\prime}
\end{array}\right) \stackrel{5.3}{=} \mathbf{s} C=\left(\begin{array}{cc}
1 & 0 \\
0 & 1
\end{array}\right) \eta, \quad \mathbf{s} c_{+}=0, \quad \mathbf{s} c_{-}=2 \eta,
\end{aligned}
$$

where $\eta$ is a Bosonic ghost-for-ghost. Nilpotency imposes $\mathbf{s} \eta=0$.

REMARK: If one identifies $\delta \leftrightarrow \mu \mathbf{s}, \widetilde{\delta} \leftrightarrow \widetilde{\mu} \mathbf{s}$, and $\Xi \leftrightarrow \mu C$, where $\mu$ and $\widetilde{\mu}$ are Fermionic parameters, then one should identify $\xi_{\mp} \leftrightarrow \mu c_{ \pm}$and $\zeta \leftrightarrow \mu \widetilde{\mu} \eta$.

In the non-minimal sector, the antighost $\bar{C}$ and the Lagrange multiplier $\Pi$ are

$$
\bar{C}=\left(\begin{array}{cc}
\bar{c} & 0 \\
0 & \bar{c}^{\prime}
\end{array}\right), \quad \Pi=\left(\begin{array}{cc}
\pi & 0 \\
0 & \pi^{\prime}
\end{array}\right) .
$$

One also has to introduce an antighost-for-ghost $\bar{\eta}$ and a Lagrange-multiplier-for-ghost $\bar{\pi}$. Moreover, there are an extra ghost $\widetilde{\eta}$ and an extra Lagrange multiplier $\tilde{\pi}$. And finally, all the fields have corresponding antifields.

A proper stage-one reducible master action $S$ is

$$
S=S_{0}+\operatorname{tr}\left(-X^{1 *} \mathbf{s} X_{1}-C^{*} \mathbf{s} C+\bar{C}^{*} \Pi\right)+\bar{\eta}^{*} \bar{\pi}+\widetilde{\eta}^{*} \tilde{\pi} .
$$

A suitable gauge-fermion $\Psi$ can be chosen on the form

$$
\Psi=\operatorname{tr}(\bar{C} \chi)+\bar{\eta} \operatorname{tr}(\Gamma C)+\operatorname{tr}(\bar{C} \Gamma) \widetilde{\eta}
$$

\footnotetext{
${ }^{1}$ We should mention Ref. [24] that also applies the BV recipe to the Connes-Lott 2-point model. (See Ref. 25] for a review of Ref. [24.) The method of Ref. [24] (implicitly) requires that all higher-stage fields should be $2 \times 2$ matrix-valued, and as a consequence, ends up with infinitely many reducibility stages by alternatingly overshooting and undershooting the single gauge-symmetry similar to the alternating series $1-2+2-2+2 \ldots$ Such infinite tower of fields is ill-defined and plagued with anomalies, i.e., the resulting partition function $\mathcal{Z}$ will depend on the choice of the gauge-fixing condition. It would be out of scope to show this in detail here, but the quickest argument is probably to notice that the final formula for the gauge-fixed action (after the infinitely many higher-stage fields have been heuristically integrated out; see formula (4.8) in Ref. [24], or equivalently, formula (14) in Ref. 25]) contains two Faddeev-Popov ghost-antighost pairs but only one independent gaugecondition. Recall that in the usual Faddeev-Popov approach, the number of gauge-conditions must precisely match the number of ghost-antighost pairs. Arguments along these lines show that the method of Ref. 24] will depend on the gauge-fixing choice, and the method therefore produces a useless result. We shall here avoid the same ill-fate by allowing for $1 \times 1$ matrix-valued stage-one fields.
} 
The fixed one-dimensional Fermionic vector $n^{1}$ from eq. (6.3) can be chosen as

$$
n^{1}=\left(\begin{array}{cc}
0 & e^{i \theta} \\
e^{-i \theta} & 0
\end{array}\right),
$$

where $\theta$ is an angle. The Lorenz type gauge condition $\chi$ reads

$$
\chi=\left[n^{1}, X_{1}\right]+\alpha \Gamma \Pi=2 \operatorname{Re}\left(H e^{-i \theta}\right) \mathbf{1}+\alpha \Gamma \Pi,
$$

where $\alpha$ is a gauge-fixing parameter. Singular (i.e., delta-function-type) gauge-fixing corresponds to $\alpha=0$, while Gaussian-type gauge-fixing corresponds to $\alpha \neq 0$. Hence the gaugefermion $\Psi$ from eq. (9.17) takes the form

$$
\Psi=\bar{c}_{+}\left[2 \operatorname{Re}\left(H e^{-i \theta}\right)+\frac{\alpha}{2} \pi_{-}\right]+\bar{\eta} c_{-}+\bar{c}_{-}\left[\widetilde{\eta}+\frac{\alpha}{2} \pi_{+}\right]
$$

where $\bar{c}_{ \pm}:=\bar{c} \pm \bar{c}^{\prime}$ and $\pi_{ \pm}:=\pi \pm \pi^{\prime}$

The antifields $\Phi^{*}=\partial \Psi / \partial \Phi$ become

$$
X^{1 *}=\left[\bar{C}, n^{1}\right], \quad C^{*}=\bar{\eta} \Gamma, \quad \bar{C}^{*}=\chi+\Gamma \widetilde{\eta}, \quad \Pi^{*}=\alpha \bar{C} \Gamma, \quad \bar{\eta}^{*}=c_{-}, \quad \tilde{\eta}^{*}=\bar{c}_{-},
$$

and all the remaining antifields $\eta^{*}, \bar{\pi}^{*}$, and $\tilde{\pi}^{*}$ are zero.

The gauge-fixed stage-one reducible action reads

$$
\left.S\right|_{\Phi^{*}=\partial \Psi / \partial \Phi}=S_{0}+\bar{c}_{+} 2 \operatorname{Im}\left(H e^{-i \theta}\right) c_{+}-2 \bar{\eta} \eta+\left[2 \operatorname{Re}\left(H e^{-i \theta}\right)+\frac{\alpha}{2} \pi_{-}\right] \pi_{-}+\left[\widetilde{\eta}+\frac{\alpha}{2} \pi_{+}\right] \pi_{+}+c_{-} \bar{\pi}+\bar{c}_{-} \tilde{\pi} .
$$

If one integrates over $\bar{\eta}, \eta, \tilde{\eta}, \pi_{+}, c_{-}, \bar{\pi}, \bar{c}_{-}$, and $\tilde{\pi}$ in the path integral, one arrives at the standard gauge-fixed stage-zero irreducible action

$$
\begin{array}{cccccc}
\left.S\right|_{\Phi^{*}=\partial \Psi / \partial \Phi} & \sim & S_{0} & + & \bar{c}_{+} 2 \operatorname{Im}\left(H e^{-i \theta}\right) c_{+} \\
\text {Gauge-fixed } & & \text { Original } & \text { Faddeev-Popov } & {\left[2 \operatorname{Re}\left(H e^{-i \theta}\right)+\frac{\alpha}{2} \pi_{-}\right]} & \pi_{-}, \\
\text {action } & & \text { action } & & \text { Gauge } & \text { Lagr. } \\
\text { action matrix } & & \text { cond. } & \text { mult. }
\end{array}
$$

with the remaining field content $H, c_{+}, \bar{c}_{+}$, and $\pi_{-}$. The Lagrange multiplier $\pi_{-}$gauge-fixes in the singular limit $\alpha=0$ the Higgs field $H$ to two opposite values $H= \pm|H| e^{i \theta}$. Here we encounter a technical (as opposed to a fundamental) Gribov ambiguity, since our simple type of gauge condition $\chi$ picks a line through the origin, which always will intersect the gauge orbit (=circle) in precisely two opposite points. (Clearly, at the fundamental level, one should just find a gauge condition that picks a half-line instead, although we shall not implement this in practice here, since it is anyway not needed.)

\section{Conclusions}

- We have, first of all, seen that the Batalin-Vilkovisky formalism [13] is a useful tool to gauge-fix matrix models, or non-commutative field theories, since such theories may exhibit reducible gauge symmetries. 
- We have for the first time shown how to successfully treat the Connes-Lott model [16] within the reducible Batalin-Vilkovisky framework [14], cf. Section 9.

- When considering matrix models one inevitable faces Itzykson-Zuber-like integrals. We have for the first time explicitly demonstrated the localization mechanism for the $U\left(N_{0} \mid N_{1}\right)$ Harish-Chandra-Itzykson-Zuber (HCIZ) integral [26, 27], cf. Appendix A. By the word explicitly, we mean, in particular, that we do not rely on the Duistermaat-Heckman Localization Theorem [28].

Acknowledgement: K.B. would like to thank Igor Batalin for discussions, and both the University of Vienna and the Erwin Schrödinger Institute for warm hospitality. The work of K.B. is supported by the Ministry of Education of the Czech Republic under the project MSM 0021622409.

\section{A HCIZ Integrals and Localization}

Let $\mathcal{A}=\operatorname{End}(V)$ be the algebra of endomorphisms in a $\left(N_{0} \mid N_{1}\right)$ super vector space $V$ of dimension $N=N_{0}+N_{1}$. Consider the Harish-Chandra-Itzykson-Zuber (HCIZ) integral [26, 27]

$$
\operatorname{HCIZ}(A, B)=\int_{U \in U(V)} \rho(U) d U e^{\frac{i}{\hbar} S_{0}}, \quad S_{0}=\operatorname{tr} L_{0}, \quad L_{0}=A U B U^{\dagger},
$$

where the integration variable $U \in U\left(N_{0} \mid N_{1}\right) \equiv U(V) \subseteq \mathcal{A}$ is a unitary endomorphism, $\varepsilon(U)=0$, and where $A, B \in \mathcal{A}$ are two fixed Bosonic Hermitian matrices, $\varepsilon(A)=0=\varepsilon(B)$. This integral is, e.g., of great importance in solving two-matrix-models. Let us choose a basis for $V$. The Haar measure is

$$
\int_{U \in U(V)} \rho(U) d U \ldots \sim \int_{U \in \operatorname{End}(V)}[d U]\left[d U^{\dagger}\right] \delta\left(U^{\dagger} U-\mathbf{1}\right) \ldots \sim \int_{U \in \operatorname{End}(V)}[d U]\left[d U^{\dagger}\right][d \Pi] e^{\frac{i}{\hbar} \operatorname{tr}\left(\left(U^{\dagger} U-\mathbf{1}\right) \Pi\right)} \ldots
$$

where $\Pi \in \mathcal{A}$ is an Bosonic Hermitian matrix that plays the rôle of Lagrange multiplier for the unitarity constraint $U^{\dagger} U=\mathbf{1}$. The Haar measure is invariant under the left-right action of $U(V)$,

$$
U \longrightarrow U_{L} U U_{R}, \quad \Pi \longrightarrow U_{R}^{\dagger} \Pi U_{R}, \quad U_{L}, U_{R} \in U(V), \quad U \in \operatorname{End}(V) .
$$

Hence we can (and will) assume without loss of generality that the fixed matrices $A$ and $B$ are both diagonal matrices

$$
A=\operatorname{diag}\left(\lambda_{1}, \lambda_{2}, \ldots, \lambda_{N}\right), \quad B=\operatorname{diag}\left(\mu_{1}, \mu_{2}, \ldots, \mu_{N}\right),
$$

with Grassmann-parity $\varepsilon\left(\lambda_{a}\right)=0=\varepsilon\left(\mu_{a}\right), a \in\{1,2, \ldots, N\}$. In particular, $[A, B]=0$. We shall furthermore assume that the eigenvalues $\lambda_{1}, \lambda_{2}, \ldots, \lambda_{N}$ of the matrix $A$ are different, and similarly, that the eigenvalues $\mu_{1}, \mu_{2}, \ldots, \mu_{N}$ of the matrix $B$ are different. We want to prove a superversion of the Harish-Chandra-Itzykson-Zuber formula [29, 30]

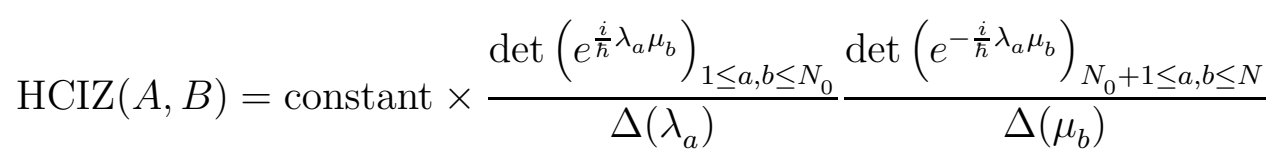


up to an overall numerical factor, which we ignore, since it is often irrelevant in physics applications. The formula (A.5) coincides with the one-loop approximation of the asymptotic steepest decent expansion for $\hbar \rightarrow 0$, cf. Ref. [31] and Ref. [32]. Our goal with this Appendix A is to provide a fully explicit localization argument that the one-loop approximation is the exact result. (In particular, we shall not rely on the Duistermaat-Heckman Localization Theorem 28], although our method is in principle equivalent. Beware that many articles, that claim to use Duistermaat-Heckman Theorem to prove localization, do actually not show that the assumptions in the Duistermaat-Heckman Theorem are fulfilled, and hence give incomplete localization arguments.) The original derivations in Ref. [29] and Ref. [30] of formula (A.5) use superversions of the heat equation method and the Gelfand-Tzetlin coordinate approach, respectively.

\section{A.1 Instantons/Classical Solutions}

An infinitesimal variation $\delta S_{0}$ of the original action $S_{0}=\operatorname{tr}\left(A U B U^{-1}\right)$ reads

$$
\delta S_{0}=\operatorname{tr}\left(E U^{-1} \delta U\right)
$$

with classical equations of motion

$$
E:=[B, H], \quad H:=U^{-1} A U .
$$

The classical equations of motion $E \approx 0$ implies that $H$ is diagonal, i.e., there exists a permutation $\sigma \in S_{N}$ such that

$$
H \approx \operatorname{diag}\left(\lambda_{\sigma(1)}, \lambda_{\sigma(2)}, \ldots, \lambda_{\sigma(N)}\right) \quad \Leftrightarrow \quad\left(\lambda_{a}-\lambda_{\sigma(b)}\right) U_{b}^{a} \approx 0,
$$

where " $\approx$ " means equality modulo classical equations of motion. Thus the matrix $U$ can at most have one non-zero entry $U^{a}{ }_{\sigma(a)}$ in each row " $a$ " (and similarly at most one non-zero entry in each column). On the other hand, to ensure that the matrix $U$ is invertible, all the entries of the form $U_{\sigma(a)}^{a}$ must be non-zero and Bosonic. This is precisely possible if the permutation $\sigma \in S_{N}$ does not mix Bosonic and Fermionic directions in $V$, i.e., $\sigma \in S_{N_{0}} \times S_{N_{1}}$. Let

$$
S_{N_{0}} \times S_{N_{1}} \ni \sigma \mapsto P_{\sigma} \in U\left(N_{0} \mid N_{1}\right) \equiv U(V) \subseteq \mathcal{A}
$$

denote the canonical embedding $S_{N_{0}} \times S_{N_{1}} \rightarrow U\left(N_{0} \mid N_{1}\right)$. The full classical solution for $U$ is a permutation matrix $P_{\sigma}$ times an element $e^{i \Xi}$ of the Cartan torus,

$$
U \approx P_{\sigma} e^{i \Xi}, \quad \sigma \in S_{N_{0}} \times S_{N_{1}}, \quad \Xi=\operatorname{diag}\left(\xi_{1}, \xi_{2}, \ldots, \xi_{N}\right) .
$$

The stationary surface of classical $U$-solutions is a disjoint union of instanton sectors, which are labelled by the permutations $\sigma \in S_{N_{0}} \times S_{N_{1}}$.

The original action $S_{0}$ has a $U(1)^{N} \times U(1)^{N}$ gauge symmetry corresponding to the left and the right Cartan torus,

$$
U \rightarrow e^{i \Xi^{\prime}} U e^{i \Xi}, \quad \Xi=\operatorname{diag}\left(\xi_{1}, \xi_{2}, \ldots, \xi_{N}\right), \quad \Xi^{\prime}=\operatorname{diag}\left(\xi_{1}^{\prime}, \xi_{2}^{\prime}, \ldots, \xi_{N}^{\prime}\right) .
$$

Since the gauge group $U(1)^{N} \times U(1)^{N}$ is compact, gauge-fixing is actually not necessary, and we shall ignore it. 


\section{A.2 A Fermionic Symmetry "s"}

In anticipation of at least one Vandermonde determinant in the final formula (A.5), let us consider the partition function

$$
\mathcal{Z}_{1}=\operatorname{HCIZ}(A, B) \Delta\left(\mu_{b}\right)=\int_{U \in \operatorname{End}(V)}[d U]\left[d U^{\dagger}\right][d \Pi][d C] e^{\frac{i}{\hbar} S_{1}}
$$

where

$$
S_{1}=\operatorname{tr} L_{1}, \quad L_{1}=H B+\frac{1}{2} C[B, C]+\left(U^{\dagger} U-\mathbf{1}\right) \Pi, \quad H:=U^{\dagger} A U
$$

The first, second, and third term in $L_{1}$ implements the original HCIZ action $S_{0}$, the Vandermonde determinant $\Delta\left(\mu_{b}\right)$, and the unitarity constraint $U^{\dagger} U=\mathbf{1}$, respectively. The $C \in \mathcal{A}$ is an (anti)Hermitian and off-diagonal Fermionic matrix. In particular, its diagonal entries $C^{a}{ }_{a}=0$ are zeroes, $1 \leq a \leq N$. (One should mention that the Gaussian Bosonic $C^{a}{ }_{b}$-integrations in eq. (A.12) are defined via analytic continuation, i.e., one should integrate along a straight line through the origin in the complex $C^{a}{ }_{b}$-plane, in such a way that the integrand becomes exponentially damped.)

To show that the integral $\mathcal{Z}_{1}$ localizes on the classical solutions (A.8), one uses a divergence-free Grassmann-odd left vector field "s",

$$
\begin{aligned}
(-1)^{\varepsilon_{a}} \mathbf{s}\left(U^{a}{ }_{c}\right) \equiv(\mathbf{s} U)^{a}{ }_{c}={U^{a}{ }_{b} C^{b}{ }_{c},}, & (-1)^{\varepsilon_{a}} \mathbf{s}\left(C^{a}{ }_{b}\right) \equiv(\mathbf{s} C)^{a}{ }_{b}=H^{\prime a}{ }_{b}:=\left\{\begin{array}{rll}
H^{a}{ }_{b} & \text { for } & a \neq b, \\
0 & \text { for } & a=b, \\
&
\end{array}\right. \\
(-1)^{\varepsilon_{a}} \mathbf{s}\left(U^{\dagger a}{ }_{c}\right) \equiv\left(\mathbf{s} U^{\dagger}\right)^{a}{ }_{c}=-C^{a}{ }_{b} U^{\dagger}{ }_{c}, & (-1)^{\varepsilon_{a}} \mathbf{s}\left(\Pi^{a}{ }_{b}\right) \equiv(\mathbf{s} \Pi)^{a}{ }_{b}=[\Pi, C]_{b}^{a} .
\end{aligned}
$$

For a review of localization techniques, see, e.g., Ref. [33]. The left vector field "s" is by definition a linear derivation $\mathbf{s}(f g)=(\mathbf{s} f) g+(-1)^{\varepsilon_{f}} f(\mathbf{s} g)$. The definition (A.14) implies the following compact formulas

$$
\mathbf{s} U=U C, \quad \mathbf{s} U^{\dagger}=-C U^{\dagger}, \quad \mathbf{s} C=H^{\prime}, \quad \mathbf{s} H=[H, C], \quad \mathbf{s} \Pi=[\Pi, C],
$$

where $H^{\prime}$ denotes the $H$-matrix with zeroes in the diagonal. Now it turns out that the $S_{1}$ action (A.13) is invariant under the Grassmann-odd s vector field

$\mathbf{s} S_{1}=\operatorname{tr}\left(B \mathbf{s} H-C[B, \mathbf{s} C]+U \Pi \mathbf{s} U^{\dagger}+\Pi U^{\dagger} \mathbf{s} U+\left(U^{\dagger} U-\mathbf{1}\right) \mathbf{s} \Pi\right)=\operatorname{tr}\left(B[H, C]-C\left[B, H^{\prime}\right]\right)=0$.

\section{A.3 Cohomology of $s$}

The divergence $\operatorname{div}(\mathbf{s})$ of the Fermionic vector field $\mathbf{s}$ vanishes

$$
\operatorname{div}(\mathbf{s})=\frac{\overrightarrow{\partial^{\ell}}}{\partial U^{a}{ }_{b}} \mathbf{s}\left(U_{b}^{a}\right)+\frac{\overrightarrow{\partial^{\ell}}}{\partial U^{\dagger a}{ }_{b}} \mathbf{s}\left(U_{b}^{\dagger a}\right)+\frac{\overrightarrow{\partial^{\ell}}}{\partial C_{b}^{a}} \mathbf{s}\left(C_{b}^{a}{ }_{b}\right)+\frac{\overrightarrow{\partial^{\ell}}}{\partial \Pi_{b}^{a}} \mathbf{s}\left(\Pi_{b}^{a}{ }_{b}\right)=0
$$

cf. definition (A.14). (The underlying reason for zero divergence is the right invariance of the Haar measure $\rho(U) d U$.) Integration by part shows that an integral $\int_{U \in U(V)} \rho(U) d U[d C] \mathbf{s} f=0$ 
over an s-exact quantity $\mathbf{s} f$ is zero, if there are no boundary contributions. Here $f=f(U, C)$ is a function. (There are never boundary contributions from Fermionic integrations, nor from Bosonic $U^{a}{ }_{b}$-integrations, which are compact directions. Boundary terms can only arise from Bosonic $C^{a}{ }_{b}$-integrations.) Perhaps surprisingly, the pertinent Fermionic "s" transformation (A.15) needed for the localization argument is not the BRST operator. Furthermore, it turns out that "s" is not nilpotent. (One of our initial motivations was to investigate whether "s" and the BRST operator would coincide, or not, and whether "s" would be nilpotent, or not.) In general, the non-nilpotency implies, among other things, that an s-exact quantity is not necessarily s-closed, and that a product of s-exact quantities is not necessarily s-exact nor $\mathbf{s}$-closed. Nevertheless, the square $\mathbf{s}^{2}$ is still a linear derivation, $\mathbf{s}^{2}(f g)=\left(\mathbf{s}^{2} f\right) g+f\left(\mathbf{s}^{2} g\right)$, with

$$
\begin{array}{rlr}
\mathbf{s}^{2} U=U\left(C^{2}+H^{\prime}\right), & \mathbf{s}^{2} H=\left[H, C^{2}+H^{\prime}\right], & \mathbf{s}^{2} C=[H, C]^{\prime}, \\
\mathbf{s}^{2} U^{\dagger}=-\left(C^{2}+H^{\prime}\right) U^{\dagger}, & \mathbf{s}^{2} \Pi=\left[\Pi, C^{2}+H^{\prime}\right] . &
\end{array}
$$

Therefore we will restrict ourselves to consider the subalgebra of integrands $f=f(U, C)$ with $\mathbf{s}^{2} f=0$. In particular, we will consider a Fermionic function $\psi=\psi(U, C)$ given by

$$
\begin{gathered}
\psi:=\operatorname{tr}(H C)=\operatorname{tr}\left(H^{\prime} C\right), \quad \mathbf{s} \psi=\operatorname{tr}\left(H^{\prime} \mathbf{s} C-C \mathbf{s} H\right)=\operatorname{tr}\left(H^{\prime} H^{\prime}-C[H, C]\right), \\
\mathbf{s}^{2} \psi=\operatorname{tr}\left(H^{\prime} \mathbf{s}^{2} C+C \mathbf{s}^{2} H\right)=\operatorname{tr}\left(H^{\prime}[H, C]+C\left[H, C^{2}+H^{\prime}\right]\right)=0 .
\end{gathered}
$$

The above cohomological consideration shows that the partition function

$$
\mathcal{Z}^{(t)}=\int_{U \in \operatorname{End}(V)}[d U]\left[d U^{\dagger}\right][d \Pi][d C] e^{\frac{i}{\hbar} S^{(t)}}
$$

with action

$$
S^{(t)}=S_{1}-\frac{1}{2 t^{2}} \mathbf{s} \psi=\operatorname{tr}\left(H B-\frac{1}{2 t^{2}} H^{\prime} H^{\prime}+\frac{1}{2} C\left[B+t^{-2} H, C\right]+\left(U^{\dagger} U-\mathbf{1}\right) \Pi\right)
$$

cannot depend on the parameter $t$, because $\mathbf{s} S_{1}=0$ and $\mathbf{s}^{2} \psi=0$. In the limit $t \rightarrow \infty$, the partition function $\lim _{t \rightarrow \infty} \mathcal{Z}^{(t)}=\mathcal{Z}_{1}$ is just the sought-for integral (A.12). (For each Bosonic Gaussian $C^{a}{ }_{b}$-integration, one might have to adjust the $C^{a}{ }_{b}$-integration contour as a function of $t$ and $U$ to ensure that the $C^{a}{ }_{b}$-integral remains exponentially damped in the integral (A.21). The value of the Gaussian $C^{a}{ }_{b}$-integral is unchanged under such shift of $C^{a}{ }_{b}$-integration contour.)

Often in the literature, one only provides an implicit existence argument that a pertinent Fermion $\psi$ with $\mathbf{s}^{2} \psi=0$ exists. Here we actually have an explicit formula (A.19) for $\psi$.

\section{A.4 Localization}

Let us scale the off-diagonal Fermionic integration variables $C \rightarrow t C$ with the number $t$. This produces a Jacobian factor

$$
\prod_{1 \leq a \neq b \leq N} t^{\left[(-1)^{\varepsilon\left(C^{a}{ }_{b}\right)}\right]}=\prod_{1 \leq a \neq b \leq N} t^{\left[-(-1)^{\varepsilon_{a}+\varepsilon_{b}}\right]}=t^{2 N_{0} N_{1}-N_{0}\left(N_{0}-1\right)-N_{1}\left(N_{1}-1\right)} .
$$

Recall that for an arbitrary complex supernumber $z$ of Grassmann-parity $\varepsilon(z)$, one has

$$
\lim _{ \pm t \rightarrow 0^{+}} t^{-2\left[(-1)^{\varepsilon(z)}\right]} \exp \left[-\frac{z \bar{z}}{2 t^{2}}\right]=\left\{\begin{array}{ccc}
2 \pi \delta^{2}(z) & \text { for } & \varepsilon(z)=0 \\
-\frac{1}{2} z \bar{z} & \text { for } & \varepsilon(z)=1
\end{array}\right\} \sim \delta^{2}(z)
$$


where we have suppress an overall numerical factor in the last expression of eq. (A.24). (In detail, the limit notation $\pm t \rightarrow 0^{+}$in eq. (A.24) is supposed to mean that the limit should be performed in such a way that $|\operatorname{Im}(t)|<|R e(t)|$ for Bosonic z.) One now let $H_{b}^{\prime a}$ in the $S^{(t)}$ action (A.22) play the rôle of $z$. (This is possible since $H^{\prime}$ is Hermitian, as a result of the matrix $A$ being Hermitian.) Adapting eq. (A.24) to the oscillatory $\mathcal{Z}^{(t)}$ integral (A.21), one is interested in the limit $\pm e^{-\frac{i \pi}{4}} t \rightarrow 0^{+}$.

$$
\lim _{ \pm e^{-\frac{i \pi}{4}} t \rightarrow 0^{+}} \mathcal{Z}^{(t)} \sim \int_{U \in \operatorname{End}(V)}[d U]\left[d U^{\dagger}\right][d C] e^{\frac{i}{\hbar} \operatorname{tr}\left(H B+\frac{1}{2} C[H, C]\right)} \delta\left(H^{\prime}\right) \delta\left(U^{\dagger} U-\mathbf{1}\right),
$$

Equation (A.25) shows that the integral localizes on the constraint $H^{\prime} \approx 0$, which, in turn, is just the stationary surface (A.10)! Hence, in order to evaluate the integral (A.25), it is enough to consider an infinitesimally small tubular $U$-neighborhood of the stationary surface (A.10). One must sum over all possible instanton sectors labelled by $\sigma \in S_{N_{0}} \times S_{N_{1}}$. For a given permutation $\sigma \in S_{N_{0}} \times S_{N_{1}}$, one may hence parametrize the $U$-variable as

$$
(\Xi, K) \longrightarrow U=P_{\sigma} e^{i \Xi} e^{K}=e^{i P_{\sigma} \Xi P_{\sigma}} P_{\sigma} e^{K},
$$

where $\Xi$ is a real, diagonal matrix, and where $K \in \mathcal{A}$ is an off-diagonal Bosonic matrix, which may be taken to be infinitesimally small. One calculates

$$
H=U^{-1} A U=e^{-K} P_{\sigma} A P_{\sigma} e^{K}=P_{\sigma} A P_{\sigma}+\left[P_{\sigma} A P_{\sigma}, K\right]+\mathcal{O}\left(K^{2}\right) .
$$

The Cartan torus $e^{i \Xi}$ in eqs. (A.10) and (A.26) just reflects a compact $U(1)^{N}$ gauge symmetry. The integration over the diagonal/gauge directions $\Xi$ therefore yields the volume vol $\left(U(1)^{N}\right)=$ $(2 \pi)^{N}$ of the Cartan torus $U(1)^{N}$, which we ignore, since we are not interested in overall numerical factors. The integral (A.25) becomes

$$
\begin{aligned}
\lim _{ \pm e^{-\frac{i \pi}{4}} t \rightarrow 0^{+}} \mathcal{Z}^{(t)} & \sim \sum_{\sigma \in S_{N_{0}} \times S_{N_{1}}} \int[d K]\left[d K^{\dagger}\right][d C] e^{\frac{i}{\hbar} \operatorname{tr}\left(P_{\sigma} A P_{\sigma} B+\frac{1}{2} C\left[P_{\sigma} A P_{\sigma}, C\right]\right)} \delta\left(\left[P_{\sigma} A P_{\sigma}, K\right]\right) \delta\left(K^{\dagger}+K\right) \\
& =\sum_{\sigma \in S_{N_{0}} \times S_{N_{1}}} \int d K[d C] e^{\frac{i}{\hbar} \operatorname{tr}\left(P_{\sigma} A P_{\sigma} B+\frac{1}{2} C\left[P_{\sigma} A P_{\sigma}, C\right]\right)} \frac{\delta(K)}{\Delta^{2}\left(\lambda_{\sigma(a)}\right)} \\
& \sim \sum_{\sigma \in S_{N_{0}} \times S_{N_{1}}} \frac{e^{\frac{i}{\hbar}(-1)^{\varepsilon} a \lambda_{\sigma(a)} \mu_{a}}}{\Delta\left(\lambda_{\sigma(a)}\right)}=\sum_{\sigma \in S_{N_{0}} \times S_{N_{1}}}(-1)^{\sigma} \frac{e^{\frac{i}{\hbar}(-1)^{\varepsilon} a \lambda_{\sigma(a)} \mu_{a}}}{\Delta\left(\lambda_{a}\right)} \\
& =\frac{\operatorname{det}\left(e^{\frac{i}{\hbar} \lambda_{a} \mu_{b}}\right)_{1 \leq a, b \leq N_{0}} \operatorname{det}\left(e^{-\frac{i}{\hbar} \lambda_{a} \mu_{b}}\right)_{N_{0}+1 \leq a, b \leq N}}{\Delta\left(\lambda_{a}\right)}
\end{aligned}
$$

in agreement with the superversion of the Harish-Chandra-Itzykson-Zuber formula [29, 30] up to a numerical factor.

\section{References}

[1] N. Seiberg and E. Witten, JHEP 9909 (1999) 032 arXiv:hep-th/9908142].

[2] H. Grosse and R. Wulkenhaar, Commun. Math. Phys. $256 \quad$ (2005) 305 arXiv:hep-th/0401128. 
[3] H. Grosse and R. Wulkenhaar, Eur. Phys. J. C 35 (2004) 277 arXiv:hep-th/0402093].

[4] M. Disertori, R. Gurau, J. Magnen and V. Rivasseau, Phys. Lett. B 649 (2007) 95 arXiv:hep-th/0612251].

[5] V. Rivasseau, arXiv:0705.0705 [hep-th].

[6] B. Jurco, L. Moller, S. Schraml, P. Schupp and J. Wess, Eur. Phys. J. C 21 (2001) 383 arXiv:hep-th/0104153.

[7] A. Bichl, J. Grimstrup, H. Grosse, L. Popp, M. Schweda and R. Wulkenhaar, JHEP 0106 (2001) 013 arXiv:hep-th/0104097.

[8] A. de Goursac, J. C. Wallet and R. Wulkenhaar, Eur. Phys. J. C 51 (2007) 977 arXiv:hep-th/0703075.

[9] H. Grosse and M. Wohlgenannt, Eur. Phys. J. C 52 (2007) 435 arXiv:hep-th/0703169.

[10] D. N. Blaschke, H. Grosse and M. Schweda, Europhys. Lett. 79 (2007) 61002 [arXiv:0705.4205 [hep-th]].

[11] L. C. Q. Vilar, O. S. Ventura, D. G. Tedesco and V. E. R. Lemes, arXiv:0902.2956 [hep-th].

[12] D. N. Blaschke, A. Rofner, M. Schweda and R. I. P. Sedmik, arXiv:0903.4811 [hep-th].

[13] I. A. Batalin and G. A. Vilkovisky, Phys. Lett. B 102 (1981) 27.

[14] I. A. Batalin and G. A. Vilkovisky, Phys. Rev. D 28 (1983) 2567 [Erratum-ibid. D 30 (1984) 508].

[15] I. A. Batalin and K. Bering, arXiv:0911.0341 [hep-th]. To appear in Int. J. Mod. Phys. A.

[16] A. Connes and J. Lott, Nucl. Phys. Proc. Suppl. 18B (1991) 29.

[17] M. Chas and D. P. Sullivan, arXiv:math/9911159 [math.GT].

[18] B. Zwiebach, Nucl. Phys. B 390 (1993) 33 arXiv:hep-th/9206084].

[19] I. A. Batalin, K. Bering and P. H. Damgaard, Nucl. Phys. B 739 (2006) 389 arXiv:hep-th/0512131.

[20] G. Barnich, M. A. Grigoriev and M. Henneaux, JHEP 0110 (2001) 004 arXiv:hep-th/0106188.

[21] G. Barnich, F. Brandt and M. Grigoriev, Fortsch. Phys. 50 (2002) 825 arXiv:hep-th/0201139.

[22] G. Barnich, F. Brandt and M. Henneaux, Commun. Math. Phys. 174 (1995) 57 arXiv:hep-th/9405109.

[23] G. Barnich, F. Brandt and M. Grigoriev, Nucl. Phys. B 677 (2004) 503 arXiv:hep-th/0308092. 
[24] H. Hüffel, J. Math. Phys. 43 (2002) 2348 arXiv:hep-th/0109134.

[25] H. Hüffel, Acta Phys. Slov. 52 (2002) 247 arXiv:hep-th/0206048].

[26] Harish-Chandra, Am. J. Math. 79 (1957) 87; ibid 80 (1958) 241.

[27] C. Itzykson and J. B. Zuber, J. Math. Phys. 21 (1980) 411.

[28] J. J. Duistermaat and G. J. Heckman, Invent. Math. 69 (1982) 259.

[29] J. Alfaro, R. Medina and L. F. Urrutia, J. Math. Phys. 36 (1995) 3085 arXiv:hep-th/9412012].

[30] T. Guhr, Commun. Math. Phys. 176 (1996) 555.

[31] M. Stone, Nucl. Phys. B 314 (1989) 557.

[32] R. J. Szabo, Nucl. Phys. B 598 (2001) 309 arXiv:hep-th/0009237.

[33] R. J. Szabo, arXiv:hep-th/9608068. 